\title{
Chao Family Comprehensive Cancer Center
}

National Cancer Institute

\section{Source}

National Cancer Institute. Chao Family Comprehensive Cancer Center. NCI Thesaurus.

Code C39338.

The Chao Family Comprehensive Cancer Center fosters bench-to-bedside research, with programs ranging from basic science research to clinical trials. Its mandate is to translate the findings of basic research into treatments that can benefit patients. Clinical research at CFCCC focuses on breast, skin, colon, liver, oral, pancreatic, and prostate cancer, and includes prevention. The center is affiliated with the School of Medicine at the University of California, Irvine. It became an NCl-designated cancer center in 1994 and achieved comprehensive cancer center status in 1997. 